\title{
Nephrotic syndrome relapse in a boy with COVID-19
}

\author{
Takuji Enya $^{1} \oplus \cdot$ Yuichi Morimoto ${ }^{1} \cdot$ Rina Oshima $^{1} \cdot$ Kohei Miyazaki $^{1} \cdot$ Tomoki Miyazawa $^{1} \cdot$ Mitsuru Okada $^{1} \cdot$ \\ Keisuke Sugimoto ${ }^{1}$
}

Received: 20 November 2020 / Accepted: 12 February 2021 / Published online: 22 February 2021

(c) Japanese Society of Nephrology 2021

\begin{abstract}
Clinical data on coronavirus disease-19 (COVID-19) in children during the management of nephrotic syndrome (NS) is lacking. Patients on prednisolone are compromised hosts at the risk of severe infections. Some infections may induce NS relapse. We describe the clinical course of a child with NS and COVID-19. A 3-year-old boy was admitted with clinical and laboratory findings indicative of NS. Induction therapy with prednisolone $(2 \mathrm{mg} / \mathrm{kg} / \mathrm{day})$ induced complete remission. While tapering the dose, he was infected with severe acute respiratory syndrome coronavirus 2 (SARS-CoV-2). He developed a high fever and periorbital edema. Urinalysis revealed proteinuria (protein-creatinine ratio: $6.3 \mathrm{~g} / \mathrm{gCr}$ ). He was transferred to our hospital for the concurrent management of COVID-19 and NS relapse. As proteinuria worsened, the prednisolone dose was increased to $2 \mathrm{mg} / \mathrm{kg} /$ day. Proteinuria gradually improved, and remission was noted a week after initiating full-dose steroid treatment. The fever subsided after 2 days without treatment for COVID-19. Anti-SARS-CoV-2 antibody including IgG levels decreased in the early convalescent phase. To the best of our knowledge, this is the first reported case with the recurrence of NS triggered by the SARS-CoV-2 infection in Asia. SARS-CoV-2 infection may induce NS relapse. Daily administration of full-dose of prednisolone may be effective for managing the recurrence of NS associated with SARS-CoV-2 infection.
\end{abstract}

Keywords COVID-19 $\cdot$ SARS-CoV-2 $\cdot$ Nephrotic syndrome $\cdot$ Relapse $\cdot$ Child

\section{Introduction}

Since its outbreak in 2019, coronavirus disease-19 (COVID19) has spread rapidly worldwide with increasing incidence and mortality rates. COVID-19 is less commonly encountered in children than in adults [1]. Respiratory syndrome is a typical symptom with severe acute respiratory syndrome coronavirus 2 (SARS-CoV-2) infection. Limited data are available regarding the renal manifestations of COVID19 namely, urinary abnormalities such as hematuria and albuminuria in critical patients and suggest an association between COVID-19 and nephritic glomerular changes [2, 3]. Nephritis-like histology was described in postmortem samples from patients with COVID-19 [4].

There is limited information about COVID-19 in children with nephrotic syndrome (NS). Only one case that describes

Takuji Enya

enyatakuji@yahoo.co.jp

1 Department of Pediatrics, Faculty of Medicine, Kindai University, 377-2 Ohno-higashi, Osaka-Sayama 589-8511, Japan simultaneous onset of NS and COVID-19 has been reported [5]. A report from Spain described 16 patients with chronic kidney disease with mild respiratory symptoms and few radiological manifestations. Glomerular filtration worsened in three of these patients, and two patients with steroid-dependent NS relapsed [6]. Patients on prednisolone are compromised hosts at risk of severe infections [7]. Furthermore, some infections may induce NS relapse [8]. We describe the first pediatric case of NS relapse triggered by SARS-CoV-2 infection in Asia.

\section{Case report}

The clinical course of this patient is shown in Fig. 1. A 3-year-old Japanese boy was admitted to the hospital with a diagnosis of NS, was treated with prednisolone and achieved complete remission. While tapering prednisolone, polymerase chain reaction (PCR) for SARS-COV-2, performed due to close contact with an infected person, was positive. The patient was transferred to our hospital for the concurrent management of COVID-19 and NS. There was a family 


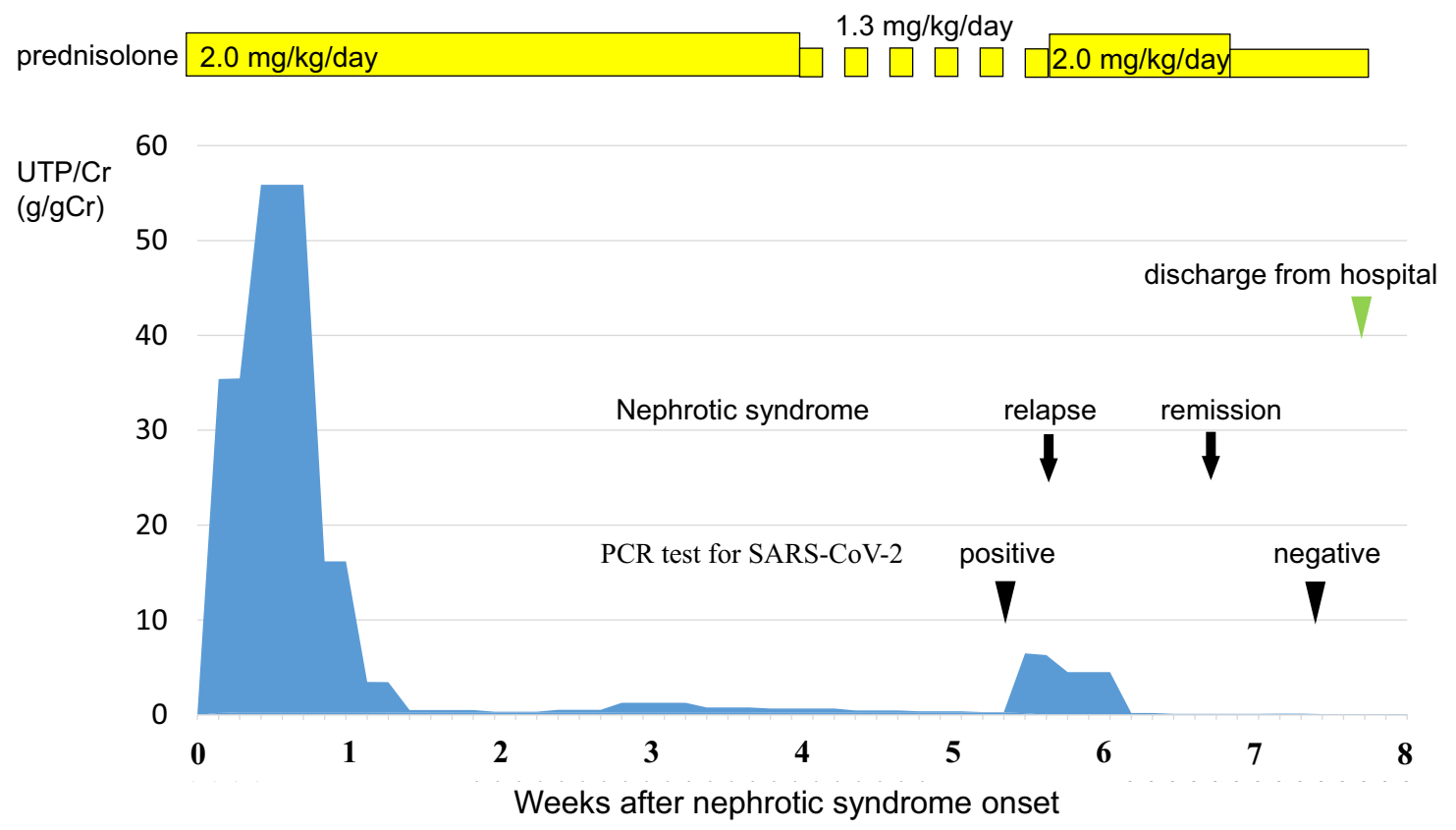

UTP/Cr : Urinary protein-creatinine ratio

Fig. 1 The clinical course of the patient

history of familial hypercholesterolemia, but not of immunodeficiency or autoimmune renal disease. On admission, no abnormalities were observed following a general physical examination, except for a high fever and periorbital edema. Physical findings were as follows: height, $89 \mathrm{~cm}$; weight, $13 \mathrm{~kg}$; blood pressure, $98 / 50 \mathrm{mmHg}$; pulse, $88 / \mathrm{min}$; percutaneous oxygen saturation, $98 \%$; and temperature, $39{ }^{\circ} \mathrm{C}$. Weight gain was $1.0 \mathrm{~kg}$. Urinalysis revealed proteinuria (protein/creatinine ratio: $6.3 \mathrm{~g} / \mathrm{gCr}$ ) but no hematuria. Hematologic examination revealed a white blood cell count of $10,700 / \mu \mathrm{L}$ (neutrophils, $90.0 \%$; lymphocytes, $7.1 \%$; monocytes, $2.6 \%$ ). Hemoglobin level and platelet count were $13.4 \mathrm{~g} / \mathrm{dL}$ and $252,000 / \mu \mathrm{L}$, respectively. Electrolytes, serum creatinine $(0.18 \mathrm{mg} / \mathrm{dL})$, and blood urea nitrogen $(4.0 \mathrm{mg} /$ $\mathrm{dL})$ were normal. The C-reactive protein level was slightly elevated $(0.37 \mathrm{mg} / \mathrm{dL})$. Aspartate and alanine aminotransferases were 29 and 11 U/L, respectively. Lactate dehydrogenase level was slightly elevated (348 U/L). Serum total protein and albumin levels were slightly decreased (6.3 and $3.5 \mathrm{~g} / \mathrm{dL}$, respectively). Serum total cholesterol, triglyceride, and low-density lipoprotein cholesterol were $606 \mathrm{mg} /$ $\mathrm{dL}$ (reference range 170-200 mg/dL), $105 \mathrm{mg} / \mathrm{dL}$ (reference range $<140 \mathrm{mg} / \mathrm{dL}$ ) and $539 \mathrm{mg} / \mathrm{dL}$ (reference range $110-140 \mathrm{mg} / \mathrm{dL}$ ), respectively. Coagulation-fibrinolysis profile was normal. Chest computed tomography (CT) examination showed no consolidations or ground-glass opacity. Fever subsided after 2 days without treatment for COVID19. The patient was treated with prednisolone at $2 \mathrm{mg} / \mathrm{kg} /$ day as proteinuria increased. The proteinuria gradually reduced, with remission occurring a week after initiating full-dose steroid treatment. After recovery from NS relapse and Covid-19, the patient was discharged from our hospital. Anti-SARS-CoV-2 antibodies including IgG levels began to decrease 2 months after the onset of SARS-CoV-2 infection.

\section{Discussion}

SARS-CoV-2 infection is spreading globally. There is less information available on clinical outcomes in children, as compared to adults. Till date, there are few reported cases of kidney involvement, including NS, in children with COVID$19[1,5]$. A retrospective case-control study was conducted among children with COVID-19 in Wuhan [9]. Eight of 260 children who were diagnosed with severe pneumonia associated with COVID-19, experienced dyspnea (87.5\%), fever $(62.5 \%)$, and cough $(62.5 \%)$ as the most common symptoms, but none of the patients developed proteinuria. Among 70 children with COVID-19 in nine New York City pediatric intensive care units, none showed renal complications such as NS, nephropathy, and nephritis [10]. According to the COVID-19 surveillance system specifically for children with chronic kidney disease from the United Kingdom Renal Registry, five children tested positive for SARS-COV-2 infection and none died [11].

It is well known that NS relapse occurs after viral respiratory infections, including adenovirus, and influenza $\mathrm{A}$ and B. Kidney injury in patients with severe COVID-19 occurs 
frequently ranging from microscopic hematuria, isolated proteinuria and azotemia, to severe acute kidney injury [2]. However, there have been no reports on concurrent pediatric nephrotic syndrome and COVID-19 that described complete clinical course including treatment. NS relapses often follow minor infections, commonly of the upper respiratory tract $[7,8]$. Daily administration of maintenance doses of prednisolone during intercurrent infections significantly reduces both relapse rates and the proportion of children with frequently relapsing nephrotic syndrome $[12,13]$. However, we increased the patient's maintenance dose of prednisolone because there was insufficient information available on clinical course of a pediatric NS patient with COVID-19. Among the four children with NS who were diagnosed with COVID-19 in Spain, two patients receiving immunosuppressants for steroid-dependent NS experienced a relapse following SARS-CoV-2 infection but showed good treatment response to prednisolone [6]. Eight children with primary or secondary immunosuppression infected with SARS-CoV-2 in Spain had respiratory symptoms and lymphopenia, five had fever, and majority showed slight radiological changes, but none developed severe disease. Most patients received antiviral treatment, and immunosuppressive treatment was decreased or discontinued after a short period of time [14]. During the second phase of COVID-19, lung involvement and lymphopenia appear or worsen, and there is a moderate increase in inflammatory markers [14]. Immunosuppressed patients may not progress to advanced disease and this could explain why most present with mild symptoms, as previously reported [14, 15]. In addition, as up to 50\% cases of COVID19 pediatric patients have abnormal chest $\mathrm{CT}$ findings, chest CT examination has become an important tool for early diagnosis of COVID-19 and for monitoring disease progression [16]. The CT manifestations in pediatric patients are diverse and lack specificity, and some pediatric patients with mild illness show no abnormalities [16]. Interestingly, our patient's lymphocyte count was significantly less than that before the SARS-CoV-2 infection, although he did not have any respiratory symptoms. This suggests that lymphopenia may not necessarily worsen disease severity in children.

The patient's serum IgG level was not measured at the time of admission because of certain difficulties. First, he was transferred to our hospital in the evening during a weekend. Second, the patient was upset and irritable during hospitalization, he was crying profusely, and tried to bite the staff while attempting to collect blood samples. In our COVID-19 meeting, we decided not to collect more blood samples for tests unless his condition warranted it, as our medical staff was exposed to risk of infection. Therefore, the patient only underwent two blood tests: on admission and before discharge from the hospital. The child was not administered intravenous immunoglobulin during hospitalization. Virus-specific IgG levels in most COVID-19 patients declined during the early convalescent phase [17]. We performed an immunoassay for the in vitro qualitative detection of antibodies including IgG to SARS-CoV-2 in human serum and plasma (Elecsys ${ }^{\circledR}$, Roche diagnostics, Rotkreuz, Switzerland). The result is reported as either reactive or non-reactive as well as in the form of a cutoff index (COI) with a finding of $<1.0$ and $\geq 1.0$ interpreted as negative and positive, respectively. The anti-SARS-CoV-2 antibody levels in our patient increased from 2.6 to 7.5 for 2 months after the onset of SARS-CoV-2 infection. Subsequently, antibody levels began to decline from 7.5 to 3.1 for the next 2 months. To the best of our knowledge, this is the first case report that highlights the clinical course of NS following SARS-COV-2 infection. No treatment for SARS-CoV-2 infection has been established yet. Systemic low or high dose of corticosteroids was associated with lower 28-day all-cause mortality among critically ill patients with COVID-19 in a metaanalysis study [18]. In addition, application of corticosteroids ( $\leq 2 \mathrm{mg} / \mathrm{kg} /$ day) could inhibit Interleukin-6 production and be effective when used in a higher dose in general type patients. This study also showed that the proper use of corticosteroids did not delay virus clearance [19]. We opted to increase the maintenance dose to full dose of prednisone for the control of proteinuria, as we anticipated that the NS relapse may worsen because of the concurrent viral infection. Considering the patient's general condition and normal coagulation profile, we believed that the benefits of full-dose steroid administration would outweigh the associated risks. Fortunately, the oral full-dose prednisolone administration was effective in controlling the NS relapse without further organ damage or clinical worsening in our patient. Daily administration of full-dose prednisolone may be effective for managing NS relapse associated with SARS-CoV-2 infection. Therefore, we concluded that a proper dose of prednisolone is required and could manage the recurrence of NS according to the NS guideline without having a significant risk. Further studies are needed to determine the guidelines for concurrent management of COVID-19 and NS in the near future.

\section{Compliance with ethical standards}

Conflict of interest The authors declare that they have no conflicts of interest.

Informed consent Informed consent was obtained from the patient's parents.

\section{References}

1. Safadi MAP. The intriguing features of COVID-19 in children and its impact on the pandemic. J Pediatr. 2020;96:265-8. 
2. Almeida FJ, Olmos RD, Oliveira DB, et al. Hematuria associated with SARS-CoV-2 infection in a child. Pediatr Infect Dis J. 2020;39:161.

3. Gross O, Moerer O, Weber M, Huber TB, Scheithauer S. COVID19-associated nephritis: early warning for disease severity and complications? Lancet. 2020;396:87-8.

4. Su H, Yang M, Wan C, et al. Renal histopathological analysis of 26 postmortem findings of patients with COVID-19 in China. Kidney Int. 2020;98:219-22.

5. Alvarado A, Franceschi G, Resplandor E, et al. COVID-19 associated with onset nephrotic syndrome in a pediatric patient: coincidence or related conditions? Pediatr Nephrol. 2020;27:1-3.

6. Melgosa M, Madrid A, Alvárez O, et al. SARS-CoV-2 infection in Spanish children with chronic kidney pathologies. Pediatr Nephrol. 2020;35:1521-4.

7. Zhang H, Wang Z, Dong L, Guo Y, Wu J, Zhai S. New insight into the pathogenesis of minimal change nephrotic syndrome: Role of the persistence of respiratory tract virus in immune disorders. Autoimmun Rev. 2016;15:632-7.

8. MacDonald NE, Wolfish N, McLaine P, Phipps P, Rossier E. Role of respiratory viruses in exacerbations of primary nephrotic syndrome. J Pediatr. 1986;108:378-82.

9. Wang Y, Zhu F, Wang C, et al. Children hospitalized with Severe COVID-19 in Wuhan. Pediatr Infect Dis J. 2020;39:91-4.

10. Derespina KR, Kaushik S, Plichta A, et al. Clinical manifestations and outcomes of critically ill children and adolescents with COVID-19 in New York City. J Pediatr. 2020;226:55-63.

11. Plumb L, Benoy-Deeney F, Casula A, et al. COVID-19 in children with chronic kidney disease: findings from the UK renal registry. Arch Dis Child. 2020; p. 319903.

12. Gulati A, Sinha A, Sreenivas V, Math A, Hari P, Bagga A. Daily corticosteroids reduce infection-associated relapses in frequently relapsing nephrotic syndrome: a randomized controlled trial. Clin J Am Soc Nephrol. 2011;6:63-9.

13. Abeyagunawardena AS, Trompeter RS. Increasing the dose of prednisolone during viral infections reduces the risk of relapse in nephrotic syndrome: a randomised controlled trial. Arch Dis Child. 2008;93:226-8.

14. Pérez-Martinez A, Guerra-García P, Melgosa M, et al. Clinical outcome of SARS-CoV-2 infection in immunosuppressed children in Spain. Eur J Pediatr. 2020;29:1-5.

15. D'Antiga L. Coronaviruses and immunosuppressed patients. The facts during the third epidemic. Liver Transplant. 2020;26:832-4.

16. Xia W, Shao J, Guo Y, Peng X, Li Z, Hu D. Clinical and CT features in pediatric patients with COVID-19 infection: different points from adults. Pediatr Pulmonol. 2020;55:1169-74.

17. Long QX, Tang XJ, Shi QL, et al. Clinical and immunological assessment of asymptomatic SARS-CoV-2 infections. Nat Med. 2020;26:1200-4.

18. The WHO Rapid Evidence Appraisal for COVID-19 Therapies (REACT) Working Group. Association between administration of systemic corticosteroids and mortality among critically ill patients with COVID-19 a meta-analysis. JAMA. 2020;324:1330-41.

19. Liu F, Ji C, Luo J, et al. Clinical characteristics and corticosteroids application of different clinical types in patients with corona virus disease 2019. Sci Rep. 2020;10:13689.

Publisher's Note Springer Nature remains neutral with regard to jurisdictional claims in published maps and institutional affiliations. 